•综述・

\title{
社会性昆虫级型和行为分化机制研究进展
}

\author{
张慧，刘倩，黄晓否*
}

闽台作物有害生物生态防控国家重点实验室，福建农林大学植物保护学院，福州 350002

\begin{abstract}
摘要: 社会性的出现是生物演化过程中的重要革新, 理解社会性的演化和调控机制具有重要的理论和实际意义。社会性昆虫 的个体间有着明显的级型分化和劳动分工，这有利于它们适应复杂的环境变化。理解社会性昆虫如何产生不同的形态、行为 和生活史特性，一直是进化和发育生物学的重要目标。随着测序技术的不断更新及生物信息学的快速发展，已经有众多关于 社会性昆虫级型和行为分化机制的研究报道。本文通过整理社会性昆虫研究的已有成果，从环境因素、生理调控和分子机制 等方面对社会性昆虫级型和行为分化机制相关研究进展进行了综述, 并对未来的研究方向做出了展望。根据现有证据, 社会 性昆虫所生活的生物环境(食物营养、信息素、表皮碳氢化合物)和非生物环境(温度、气候等)均能直接或间接影响社会性 昆虫级型和行为的分化; 保幼激素、蜕皮激素、类胰岛素及生物胺等内分泌激素和神经激素对社会性昆虫的级型和行为分 化也有重要的调控作用; 此外, 遗传因素、新基因等DNA序列或基因组结构上的变化以及表观遗传修饰、基因的差异表达 等基因调控机制均能不同程度地影响社会性昆虫的行为分化。本文建议加强昆虫纲其他社会性类群如半翅目蚜虫和缨翅 目蓟马等的社会性行为及其演化机制的研究，以加深对社会性昆虫起源及其行为演化的理解和认识。
\end{abstract}

关键词：社会性昆虫; 行为分化; 环境因素; 生理调控; 分子机制

张慧, 刘倩, 黄晓否 (2021) 社会性昆虫级型和行为分化机制研究进展. 生物多样性, 29, 507-516. doi: 10.17520/biods.2020224.

Zhang H, Liu Q, Huang XL (2021) Mechanisms regulating caste and behavior differentiation in social insects. Biodiversity Science, 29, 507-516. doi: 10.17520/biods.2020224.

\section{Mechanisms regulating caste and behavior differentiation in social insects}

Hui Zhang ${ }^{(\mathbb{D})}$, Qian Liu, Xiaolei Huang ${ }^{(\mathbb{D} *}$

State Key Laboratory of Ecological Pest Control for Fujian and Taiwan Crops, College of Plant Protection, Fujian Agriculture and Forestry University, Fuzhou 350002

\section{ABSTRACT}

Background \& Aims: Eusociality is a critical evolutionary innovation. Understanding the origin of eusociality and related regulating mechanisms has theoretical and practical significance to several research fields. A clear hierarchy and division of labor exists among individuals of social insects. The behavioral differentiation and extensive cooperation between castes are beneficial for their adaptation to complicated environmental changes. Understanding how social insects can produce individuals with differences in morphology, behavior and life-history characteristics is an important goal of much evolutionary and developmental biology research. With the rapid development of sequencing technology and bioinformatics, there have been many studies on the mechanisms underlying social insect behavioral differentiation. Here, we present recent advances on the environmental factors and physiological and molecular mechanisms regulating caste and behavioral differentiation in social insects by summarizing the current results of social insect studies, and propose the future research directions.

Progresses: Both biotic factors (e.g., nutrients, pheromones, cuticular hydrocarbons) and abiotic factors (e.g., temperature, climate) can directly and indirectly affect the differentiation of insect social behavior and castes. Endocrine hormones, such as juvenile hormone (JH), ecdysteroids (20E), insulin-like peptides (ILPs), and neurohormonal bioamines, also play important roles. In addition, evolutionary changes in gene sequences or genome structure, including heritable differences and novel genes, as well as gene regulatory mechanisms, such as DNA methylation and

收稿日期: 2020-05-29; 接受日期: 2020-09-01

* 通讯作者 Author for correspondence. E-mail: huangxl@fafu.edu.cn 
differential expression of genes, can also affect the caste and behavior differentiation of social insects to different degrees.

Prospects: We suggest strengthening the study of social behavior and regulating mechanisms in other social insect lineages, such as aphids and thrips, which are relatively understudied and which will improve the understanding of the origins and evolution of eusociality and social behaviors in insects.

Key words: social insect; behavioral differentiation; environmental factor; physiological regulation; molecular mechanism

典型的真社会性昆虫包括膜翅目的蜜蜂、蚂 蚁、胡蜂以及蜚蠊目的白蚁(原归于等翅目)等, 常具 有亲代照料、生殖分工和世代重叠三个最主要的特 征(Wilson, 1971; Wilson \& Hölldobler, 2005)。在外 界或种群内部选择压力的影响下, 社会性昆虫将利 他性作用于群体层面而非个体本身(Corona et al, 2016), 导致了不同个体间职能的分工, 也就是所谓 的劳动分工。社会性昆虫的劳动分工一般包括生殖 个体与非生殖个体间的生殖分工, 以及不具生殖功 能的工虫和兵虫的任务分工; 不同级型个体因分工 的不同而分化出不同的行为。常见社会性昆虫的种 群一般由王后和职虫(工虫和兵虫)阶级组成, 它们 在形态、行为和寿命等方面均有着明显不同; 许多 社会性昆虫种类的职虫阶级因承担的任务不同, 其 行为也存在分化, 如亲代照料、受食或看守巢穴等 (Robinson, 1992)。一些物种内职虫行为的分化往往 还伴随着形态上的特化, 比如在佛罗里达弓背蚁 (Camponotus floridanus)中防卫蚁巢的兵蚁要比那 些受食和照料后代的工蚁的体型大许多(Simola et al, 2016)。社会性昆虫在进化和生态上的成功主要 归因于其生活方式由独居性到社会性生活的重大 改变, 社会性昆虫级型之间的职能分工和广泛合作 有利于它们适应复杂的环境变化(Wilson, 1985, 1987)。

社会性昆虫王后与职虫间的级型分化以及不 同职虫间的行为分化一直是进化生物学研究的热 点(LeBoeuf et al, 2013; Gadagkar et al, 2019)。目前 研究最多的社会性昆虫是蜜蜂、蚂蚁、白蚁等真社 会性昆虫, 其他一些社会昆虫类群如半翅目的一些 蚜虫物种(Stern \& Foster, 1996)和缨翅目的一些蓟 马物种(Crespi, 1992), 也表现出一定程度的社会性, 如防御天敌或外来入侵者等利他社会行为, 但一般 缺乏广泛的合作或繁殖分工, 目前关于这类社会性 昆虫的研究不多, 对这两个类群行为分化的了解和 认识也较少。理解社会性昆虫如何演化出不同的形
态、行为或生活史特性，一直以来都是进化和发育 生物学的一个重要内容, 对社会性昆虫演化及其行 为分化机制的探索更是引起了广泛关注(Toth \& Robinson, 2007; Toth \& Rehan, 2017; Weitekamp et al, 2017)。

不同的社会性昆虫类群所面临的选择压力或 具有的社会性复杂程度也有所不同, 导致其级型和 行为分化的因素也具有多样性(Zayed \& Kent, 2015; Toth \& Rehan, 2017)。目前对社会性昆虫行为机制 的研究已经从最初相关环境因子的探索逐步发展 到内部生理调控及分子作用机理上。近年来, 分子 测序技术和生物信息学工具的发展快速推进了对 不同社会性昆虫类群的比较和进化基因组学研究 (Simola et al, 2013; Kapheim et al, 2015; Harrison et al, 2018), 加深了对社会行为产生和进化机制的理 解。基于对近些年代表性研究的梳理, 本文将从影 响昆虫社会行为的环境因素、生理调控和分子机制 等方面对社会性昆虫行为分化机制研究进展进行 综述。

\section{影响社会性昆虫级型和行为分化的环境因}

环境因素可以直接或间接地影响社会性昆虫 的行为和进化。这些环境因素主要分为生物因素和 非生物因素, 也就是昆虫所生活的生物环境及非生 物环境。其中生物因素主要指社会性昆虫所生活的 社会环境(如母体效应、亲缘效应等), 非生物因素包 括温度、气候等外界因子(Toth \& Rehan, 2017)。已 有证据表明，食物、王后信息素、表皮碳氢化合物、 王后年龄、温度、气候等因素均可影响社会性昆虫 级型和行为的分化。

社会性昆虫生活在一个比较复杂的社会性环 境中, 个体在发育的过程中很容易受到营养、信息 素、个体间物理接触等的影响。社会性昆虫的级型 分化可能在个体发育的早期阶段就已经发生, 如母 体对后代营养分配的不均衡 (Wheeler, 1996; 
Schwander et al, 2008; Judd et al, 2015)。营养是社会 性昆虫级型和行为分化的一个主要驱动力, 鉴于食 物通常由群体中其他成员所提供, 营养可以看作一 种社会环境因素。在蜜蜂中, 幼虫期营养物质的数 量和质量决定了它们的发育方向, 如被喂食营养丰 富的蜂王浆的幼虫会发育为蜂后, 而被喂食营养较 缺乏食物的幼虫则发育为工蜂 (Haydak, 1943; Kamakura, 2011)。年龄较大的工蜂负责外出受食, 年龄较小的工蜂则负责储存食物及喂养幼虫等巢 内事务, 而由巢内工作到外出受食的转变时间是由 社会环境和营养因素共同调控的(Toth et al, 2005; Ament et al, 2011)。除蜜蜂外, 蚂蚁、胡蜂和白蚁的 生理、行为和发育也受营养因素的调控(Cassill \& Tschinkel, 1999; Korb \& Schmidinger, 2004; Daugherty et al, 2011)。

化学信号是另一个影响社会性昆虫行为分化 的生物因素, 主要包括来自种群中其他个体的表皮 碳氢化合物和信息素。例如, 在红胡须蚁 (Pogonomyrmex barbatus)中, 执行不同任务的工蚁 所具有的碳氢化合物不同(Greene \& Gordon, 2003)。 蜜蜂警戒信息素的有效成分为乙酸异戊酯(IAA), 能够引起大多数蜜蜂的防御反应(Nouvian et al, 2018); 而由蜜蜂蜂后所释放的信息素, 活性成分主 要有反式-9-氧代-2-癸烯酸(9-ODA)、正/反式-9-羊弪 基-2-癸烯酸(+/-9-HDA)、对羟基苯甲酸甲酯( $\mathrm{HOB}) 、$ 4-羟基-3-甲氧基苯乙醇(HVA)等(Slessor et al, 1988), 能够抑制工蜂的卵巢发育, 从而使蜂后垄断了生殖 权; 若将蜂后从蜂巢中移除, 工蜂的卵巢就会有一 定程度的发育, 并可能恢复生殖能力 (Page \& Erickson, 1988; Barron \& Oldroyd, 2001)。信息素也 能够影响白蚁的级型分化, 由补充生殖蚁分泌的含 丁酸丁酯和2-甲基-1-丁醇两种活性成分的信息素 能够抑制蚁群中新的补充生殖蚁的产生, 且白蚁的 卵也能够分泌这种信息素, 以此作为吸引工蚁抚育 照料的引诱剂以及补充生殖蚁分化的抑制剂 (Matsuura et al, 2010)。此外, 信息素也会参与调节 蚂蚁的社会性行为, 如暗足弓背蚁 (Camponotus obscuripes)警报信息素的活性成分是甲酸和十一烷, 能够激活蚂蚁触角上的神经元, 从而调控激发弓背 蚁的攻击行为(Mizunami et al, 2010)。

此外, 在某些社会性昆虫物种中, 王后年龄也 是影响其级型分化的一种生物因素。罗纹须蚁
(Pogonomyrmex rugosus)蚁后年龄对后代的级型分 化有重要影响, 只有那些年龄大于两年的蚁后所产 的卵才能发育为新的蚁后(Schwander et al, 2008)。 王后年龄的影响在小红蚁(Myrmica rubra)中也有所 体现，一般年轻蚁后所产的卵中发育为工蚁的比例 会更大(Brian \& Hibble, 1964)。

非生物的环境因子也是昆虫社会性合作行为 的重要驱动力。如对瑞士阿尔卑斯山脉的 176 种社 会性程度不同的膜翅目昆虫种群的生活史和分布 进行比较研究发现, 海拔和季节长短可以影响兼性 群居物种的独居性与合作建巢行为的动态转变 (Kocher et al, 2014)。对初级社会性类群马蜂属 (Polistes)的研究表明, 其社会性的合作繁殖可能受 气候变化的影响, 合作种群的形成与短时期内较大 的温度波动有关, 且在比较温暖湿润的环境下, 合 作建巢蜂后的数量也会增多(Sheehan et al, 2015)。 温度也会影响蚂蚁的级型分化。罗纹须蚁的蚁后只 有经历一段时间低温(越冬)后产下的卵才能发育成 为新的蚁后(Schwander et al, 2008), 而台湾乳白蚁 (Coptotermes formosanus)工蚁向兵蚁的转化受温度 的影响较大, 较高的温度条件能促使产生更多的兵 蚁(Fei \& Henderson, 2002; Tarver et al, 2012)。

\section{2 社会性昆虫级型和行为分化的生理调控}

保幼激素 $(\mathrm{JH})$ 、蜕皮激素 $(20 \mathrm{E})$ 、类胰岛素 (insulin-like peptides, ILPs)及生物胺等内分泌激素 和神经激素对社会性昆虫的行为和级型分化有着 重要的调控作用。关于调控社会性昆虫劳动分工的 生理机制已经在蜜蜂中开展了广泛研究, 比如给蜜 蜂幼虫喂食不同营养的食物会导致蜂后和工蜂的 分化, 而食物对蜜蜂级型的调节是通过保幼激素水 平的变化实现的; 此外, 保幼激素还参与调节蜜蜂 和熊蜂工蜂行为的分化(Sullivan et al, 2000; Schulz et al, 2002; Amsalem et al, 2014)。在蚂蚁中亦发现了 该现象, 高的保幼激素水平能够使切叶蚁工蚁的行 为发生转变, 使其更多地由巢内活动转为外出受食 活动(Norman \& Hughes, 2016)。很多对白蚁的研究 均表明保幼激素在调控其级型分化中起到至关重 要的作用(Watanabe et al, 2014; Korb, 2015; Korb \& Belles, 2017)。如对山林原白蚁(Hodotermopsis sjostedti)的研究发现高的JH滴度与其兵蚁的分化有 关, 而补充型繁殖蚁中 $\mathrm{JH}$ 的滴度相对较低, 说明 $\mathrm{JH}$ 
可能参与了白蚁的兵蚁和繁殖蚁的分化(Cornette et $\mathrm{al}, 2008)$ 。蜕皮激素被认为与某些社会性昆虫的卵 黄蛋白合成相关(Dong et al, 2009)。对内华达古白蚁 (Zootermopsis nevadensis)的研究发现, 其头部的 $\beta$ 转化生长因子 (TGF $\beta)$ 信号与白蚁级型分化有关, 它 可以通过调节 $20 \mathrm{E}$ 和JH信号来调控白蚁兵蚁的分化 过程(Masuoka et al, 2018)。

胰岛素是一类进化十分保守的多功能性肽类 激素, 存在于众多生物体中, 昆虫体内也存在类似 结构和功能的肽类激素, 被称为 “类胰岛素肽”。昆 虫类胰岛素肽可参与调控昆虫的寿命以及代谢、生 长发育和生殖等生命活动(Wu \& Brown, 2006), 使 其成为调节社会性昆虫劳动分工的主要候选因素。 类胰岛素直接发挥的功能较少, 主要通过类胰岛素 受体以及相关信号通路来调节昆虫的各种生理过 程。在昆虫中, 以多基因家族编码的类胰岛素(ILPs) 为主, 其功能与哺乳动物的胰岛素和胰岛素样生长 因子1 (IGF1)是同源的, 是昆虫生活史的重要调控 因子(Flatt et al, 2005)。大多数被研究的膜翅目昆虫 有两种类胰岛素: ILP1和ILP2, 在结构上, ILP1类似 胰岛素样生长因子, ILP2 与胰岛素比较相似 (Chandra et al, 2018)。多数蚂蚁的级型分化是通过 发育过程中汲取营养的不平衡引起的, 这会导致幼 虫体内胰岛素水平上的差异, 使食物充足的蚁后有 着较高的胰岛素水平(Trible \& Kronauer, 2017)。最 近一项对 7 种蚂蚁的生殖蚁和非生殖蚁的脑部转录 组比较研究发现, ILP2总是在生殖蚁中高表达, 其 中毕氏卵角蚁(Ooceraea biroi)幼虫释放的信号通过 降低ILP2水平抑制成虫生殖, 使其产生亲代抚育行 为, 而增加ILP2 可以抵消幼虫的这种抑制作用 (Chandra et al, 2018)。此外, 胰岛素或其相关通路也 可参与调控蜜蜂的级型分化及工蜂的劳动分工 (Ament et al, 2008; de Azevedo \& Hartfelder, 2008; Mott \& Breed, 2012)。

生物胺类如五羟色胺 $(5-\mathrm{HT})$ 、酪胺 $(\mathrm{TA}) 、$ 章鱼 胺 $(\mathrm{OA})$ 和多巴胺 $(\mathrm{DA})$ 等作为神经调质或神经递质 存在于神经系统中, 对昆虫的生理和行为有着重要 的调控作用(Monastirioti, 1999; Scheiner et al, 2006; Wada-Katsumata et al, 2011)。五羟色胺、多巴胺以 及酪胺被证明对蚂蚁的攻击性行为有明显的影响 (Szczuka et al, 2013), 而章鱼胺系统可参与调控蚂
蚁各级型间(蚁后和工蚁)及工蚁不同型间(大、小工 蚁)攻击行为的转变(Aonuma \& Watanabe, 2012; Kamhi et al, 2015)。白蚁兵蚁中章鱼胺和酪胺的水 平要高于工蚁, 章鱼胺、酪胺水平升高可加强兵蚁 的攻击防御行为, 而工蚁经酪胺处理后也可产生防 御行为(Ishikawa et al, 2016)。在蜜蜂中, 章鱼胺可 刺激蜜蜂体内的咽侧体分泌保幼激素(Kaatz et al, 1994), 且能够调节工蜂的劳动分工、诱导受食行为 的发生, 其中受食工蜂的大脑中比亲代抚育工蜂有 更高的章鱼胺水平(Schulz et al, 2002; Reim \& Scheiner, 2014), 章鱼胺还可参与调控蜜蜂的飞舞 行为(Schulz et al, 2002; Barron et al, 2007; Reim \& Scheiner, 2014)。此外, 蜜蜂的警戒信息素可以提升 脑中五羟色胺和多巴胺的水平, 引起蜜蜂攻击和叮 刺等防御行为(Nouvian et al, 2018)。

\section{3 社会性昆虫级型和行为分化的分子机制}

随着基因组学、转录组学、甲基化检测技术、 RNAi技术、苂光定量PCR技术以及生物信息学分析 的发展, 有关社会性昆虫级型和行为分化的分子机 制也逐渐被揭示, 包括基于DNA序列和基因组结构 上的发现及基因调控机制的探索。

\subsection{DNA序列改变}

\subsection{1 遗传决定}

级型分化一直以来都是社会性昆虫分子水平 研究所聚焦的领域, 社会性昆虫各级型的分化大都 是环境诱导的基因表达变化引起，而不是遗传决定 的, 但也有级型由遗传因素决定的例子存在。关于 社会性昆虫劳动分工的遗传和基因组分析常限于 为数不多的物种, 但随着对更多物种研究的深入, 发现遗传因素对社会性昆虫劳动分工的影响比原 先预想的要普遍得多, 对社会性昆虫各级型的行 为、形态和生理方面都有一定的影响(Smith et al, 2008)。须蚁属的红胡须蚁和罗纹须蚁是遗传级型决 定(genetic caste determination, GCD)的两个特殊例 子: 两个物种在分布的重叠区域可以进行种间杂交, 最终每个种都获得一对不同的杂交谱系，谱系间杂 交产生工蚁, 谱系内交配产生蚁后(Julian et al, 2002; Cahan \& Keller, 2003; Anderson et al, 2006)。由遗传 因素引起的社会性昆虫的级型决定在其他蚂蚁和 白蚁中也有报道(Pearcy et al, 2004; Hayashi et al, 2007; Wiernasz \& Cole, 2010)。另外, 遗传因素对社 
会性昆虫工蜂(蚁)之间的行为分化也有一定的影 响。例如在蜜蜂种群内, 由于蜜蜂多次交配的特点, 群体内工蜂的遗传异质性程度很高, 而工蜂之间的 遗传差异能够影响工蜂间的劳动分工, 比如看守蜂 巢入口的工蜂与清除蜂巢内尸体的工蜂之间行为 的分化(Frumhoff \& Baker, 1988; Robinson \& Page, 1988)。对顶切叶蚁属的棘顶切叶蚁 (Acromyrmex echinatior)及游蚁属的Eciton hamatum研究发现, 同 一种群中来自不同父系的个体, 具有发展成为体型 不等的兵、工蚁的潜能, 从而产生了不同的劳动分 工(Hughes et al, 2003; Jaffé et al, 2007)。

\subsection{2 新基因}

新基因在社会性昆虫表型进化革新的过程中 起着十分重要的作用, 每一个新测序的社会性昆虫 的基因组中都存在新基因, 并且比例可能达到 10\%-30\% (Wissler et al, 2013; Sumner, 2014)。社会 性昆虫基因组中的新基因与各级型形态或行为的 特化有着紧密联系, 如对 7 种蚂蚁的比较基因组研 究发现新基因在它们的适应性进化过程中有着重 要的作用, 每个物种的基因组中含有大量物种特异 的新基因, 表明社会性昆虫在进化过程中存在新基 因快速获取的过程, 这可能与物种特异性特征的适 应性进化有关(Simola et al, 2013)。对蜜蜂进行群体 基因组学研究发现, 那些仅存在于蜜蜂中的新基因 有着显著的正选择作用, 尤其是那些在工蜂中高表 达的新基因(Harpur et al, 2014), 并且有研究表明在 蜜蜂工蜂中高表达的新基因数目是蜂后中高表达 新基因的2倍(Johnson \& Tsutsui, 2011), 说明新基因 在工蜂特征的适应性进化中扮演着重要角色。同样 的情况在初级社会性的红纸黄蜂 (Polistes canadensis) 中也有发现, 该物种级型之间 $75 \%$ 的差 异表达基因是新基因, 其中在工蜂中上调表达的新 基因占到90\% (Ferreira et al, 2013)。

\section{2 基因调控}

基因调控(如表观遗传修饰和基因差异表达)对 社会性昆虫特性的演化同样有着重要作用(Harrison et al, 2018; Marshall et al, 2019)。基因调控是在不改 变DNA序列的前提下, 通过改变昆虫生长发育相关 的基因功能或表达情况来调控昆虫的形态、行为或 生理, 进而使其产生不同的表型, 对社会性昆虫的 级型和行为分化均有着重要的影响。

\subsection{1 表观遗传修饰}

表观遗传修饰如DNA甲基化在调控社会性昆 虫表型可塑性进化的过程中扮演着重要的角色 (Weiner \& Toth, 2012)。目前关于表观遗传修饰对社 会性昆虫级型决定和行为分化的相关调控机制的 研究已有许多。如 $\mathrm{Li}$ 等(2018) 确定了欧洲熊蜂 (Bombus terrestris)中6个重要的调控DNA甲基化修 饰的基因, 并发现这些基因表现出一定的级型特异 性表达模式, 与工蜂、雄蜂相比, 蜂后有着较高的 表达水平。此外, 对蜜蜂的表观遗传分析发现, 幼 虫在不同的营养或空间等饲养条件下, 其发育相关 通路上的DNA甲基化水平也会有所不同, 进而产生 蜂后与工蜂的分化(He et al, 2017)。佛罗里达弓背蚁 和印度跳蚁(Harpegnathos saltator)在社会性程度及 等级制度上均有着很大的不同: 佛罗里达弓背蚁的 种群由蚁后以及形态和行为显著不同的大、小工蚁 组成; 而印度跳蚁的蚁后和工蚁形态相近, 且工蚁 有着发育为蚁后的可塑能力。对二者进行基因组比 较研究发现, 两种蚂蚁的DNA甲基化程度差异明显, 有着较原始社会性生活方式的印度跳蚁相对于佛 罗里达弓背蚁有着较低的DNA甲基化水平(Bonasio et al, 2010)。此外, 这两种蚂蚁各级型之间也有着明 显不同的甲基化模式, 揭示了表观遗传修饰在调控 不同级型之间的劳动分工方面起着重要作用 (Bonasio et al, 2012; Chittka et al, 2012)。

\subsection{2 基因的差异表达}

关于基因差异表达对社会性昆虫级型或行为 分化的影响已被相关研究所证实(Whitfield et al, 2003; Steller et al, 2010; Chandrasekaran et al, 2011)。 对北美散白蚁(Reticulitermes flavipes)的生殖型幼虫 与无生殖能力的兵蚁和工蚁的比较转录组学研究 发现, 二者共有 93 个基因差异表达, 其中表现为兵 蚁特异性的基因占所有差异表达基因的78\%, 这些 基因可能导致了防御型兵蚁与生殖蚁和工蚁之间 功能的分化(Wu et al, 2018)。一些蚂蚁物种的种群 内分化出形态及行为明显不同的兵蚁和工蚁, 兵蚁 体型较大, 一般负责守卫巢穴, 工蚁体型较小, 负 责外出受食。有研究发现苍白大头蚁 (Pheidole pallidula) 的兵蚁在某些情况下也能够转为受食, 而 这是通过大头蚁中受食基因(ppfor)编码的环鸟苷酸 依赖性蛋白激酶(cGMP-PKG) 所调控的。通常兵蚁 的脑部相对于受食的工蚁有着较高的环腺苷酸依 
赖性激酶活性，且该酶在二者脑中的空间分布也有 所不同。当用食物刺激兵蚁时, 其脑中的PKG表达 量会降低, 而当蚁巢需要防御时, 兵蚁中该酶的表 达量明显升高(Lucas \& Sokolowski, 2009)。另外, 对 亚社会性木蜂、熊蜂的转录组学研究表明, 种群内 个体因职能分工(生殖和亲代照料)不同, 其基因表 达模式也存在很大差异(Rehan et al, 2014; Woodard et al, 2014)。

此外, 一些比较重要的单一基因的差异表达对 不同社会性昆虫的行为分化有重要影响。例如卵黄 蛋白的前体物质卵黄原蛋白(vitellogenin, Vg), 可作 为雌性昆虫生殖活动的重要指标, 也是社会性昆虫 级型和行为分化的重要调控因子, 通常在各物种的 不同个体中表现出级型特异性的表达模式。在蜜蜂 中，通过 RNAi干扰 $V g$ 基因的表达后，工蜂由巢内 工作向外出受食行为转变的时间提早(Nelson et al, 2007; Marco Antonio et al, 2008)。通过比较红胡须蚁
中两个 $V g$ 基因的表达模式，发现 $P b \_V g 1$ 在蚁后(相 较于兵蚁)及亲代照料工蚁(相较于受食工蚁)中高 表达，而相较于亲代抚育工蚁和蚁后， $P b \_V g 2$ 在受 食工蚁中高表达，表明蚂蚁亦可通过控制 $V g$ 基因的 表达来调控其个体间行为的分化(Corona et al, 2013)。Vg基因也可参与调控白蚁的级型分化, 如内 华达古白蚁(Zootermopsis nevadensis)基因组中有4 个 $V g$ 基因拷贝，其中有 3 个在蚁后中的表达显著高 于其他级型(Terrapon et al, 2014)。

\section{4 小结与展望}

不同类群的社会性昆虫所面对的生态因素及 选择压力不同，其社会性复杂程度以及分化机制也 有所不同。前人对膜翅目蜜蜂、蚂蚁、胡蜂和蜚蠊 目白蚁的研究已经揭示了一些高级社会性昆虫的 劳动分工和行为分化的机制(表1列出了一些代表性 研究), 但对于昆虫纲其他社会性昆虫类群如半翅

表1 社会性昆虫级型和行为分化的影响因素

Table 1 Influencing factors underlying caste and behavior differentiation in social insects

\begin{tabular}{|c|c|c|c|c|}
\hline $\begin{array}{l}\text { 影响因素 } \\
\text { Influencing factors }\end{array}$ & & $\begin{array}{l}\text { 昆虫类群 } \\
\text { Insect groups }\end{array}$ & $\begin{array}{l}\text { 劳动分工类型 } \\
\text { Labour division }\end{array}$ & $\begin{array}{l}\text { 参考文献 } \\
\text { References }\end{array}$ \\
\hline $\begin{array}{l}\text { 外界环境 } \\
\text { External environment }\end{array}$ & $\begin{array}{l}\text { 营养 } \\
\text { Nutrition } \\
\text { 表皮碳氢化合物 } \\
\text { Cuticular hydrocarbons } \\
\text { 信息素 } \\
\text { Pheromone } \\
\text { 王后年龄 } \\
\text { Queen age } \\
\text { 海拔和季节 } \\
\text { Elevation and season } \\
\text { 气候 } \\
\text { Climate } \\
\text { 温度 } \\
\text { Temperature }\end{array}$ & $\begin{array}{l}\text { 西方蜜蜂 } \\
\text { Apis mellifera } \\
\text { 红胡须蚁 } \\
\text { Pogonomyrmex barbatus } \\
\text { 黄胸散白蚁 } \\
\text { Reticulitermes speratus } \\
\text { 罗纹须蚁 } \\
\text { Pogonomyrmex rugosus } \\
\text { 蜜蜂总科 } \\
\text { Apoidea } \\
\text { 马蜂属 } \\
\text { Polistes } \\
\text { 台湾乳白蚁 } \\
\text { Coptotermes formosanus }\end{array}$ & $\begin{array}{l}\text { 蜂后-工蜂 } \\
\text { Queen-worker } \\
\text { 受食-侦查 } \\
\text { Foraging-patrolling } \\
\text { 生殖蚁-工蚁 } \\
\text { Neotenics-worker } \\
\text { 蚁后-工蚁 } \\
\text { Queen-worker } \\
\text { 独居-合作筑巢 } \\
\text { Solitary-cooperative } \\
\text { 合作繁殖 } \\
\text { Cooperative breeding } \\
\text { 工蚁-兵蚁 } \\
\text { Worker-soldier }\end{array}$ & $\begin{array}{l}\text { Matsuura et al, } 2010 \\
\text { Schwander et al, } 2008 \\
\text { Kocher et al, } 2014 \\
\text { Sheehan et al, } 2015 \\
\text { Tarver et al, } 2012\end{array}$ \\
\hline $\begin{array}{l}\text { 生理调控 } \\
\text { Physiological regulation }\end{array}$ & $\begin{array}{l}\text { 保幼激素 } \\
\text { Juvenile hormone } \\
\text { 蜕皮激素 } \\
\text { Ecdysone } \\
\text { 类胰岛素 } \\
\text { Insulin-like peptides } \\
\text { 生物胺 } \\
\text { Biogenic amine }\end{array}$ & $\begin{array}{l}\text { 八刺顶切叶蚁 } \\
\text { Acromyrmex octospinosus } \\
\text { 内华达古白蚁 } \\
\text { Zootermopsis nevadensis } \\
\text { 毕氏卵角蚁 } \\
\text { Ooceraea biroi } \\
\text { 西方蜜蜂 } \\
\text { Apis mellifera }\end{array}$ & $\begin{array}{l}\text { 巢内活动-受食 } \\
\text { Nest work-foraging } \\
\text { 兵蚁-工蚁 } \\
\text { Soldier-worker } \\
\text { 工蚁-生殖蚁 } \\
\text { Worker-reproductives } \\
\text { 攻击防御 } \\
\text { Defensive aggression }\end{array}$ & $\begin{array}{l}\text { Norman \& Hughes, } 2016 \\
\text { Masuoka et al, } 2018 \\
\text { Chandra et al, } 2018 \\
\text { Nouvian et al, } 2018\end{array}$ \\
\hline $\begin{array}{l}\text { 分子调控 } \\
\text { Molecular regulation }\end{array}$ & $\begin{array}{l}\text { 遗传决定 } \\
\text { Genetic determination } \\
\text { 新基因 } \\
\text { Novel gene } \\
\text { 表观遗传修饰 } \\
\text { Epigenetic modification } \\
\text { 基因差异表达 } \\
\text { Gene differential expression }\end{array}$ & $\begin{array}{l}\text { 欶顶切叶蚁 } \\
\text { Acromyrmex echinatior } \\
\text { 西方蜜蜂 } \\
\text { Apis mellifera } \\
\text { 西方蜜蜂 } \\
\text { Apis mellifera } \\
\text { 北美散白蚁 } \\
\text { Reticulitermes flavipes }\end{array}$ & $\begin{array}{l}\text { 大工蚁-小工蚁 } \\
\text { Major-minor worker } \\
\text { 工蜂特性 } \\
\text { Worker traits } \\
\text { 蜂后-工蜂 } \\
\text { Queen-worker } \\
\text { 兵蚁-工蚁(生殖蚁) } \\
\text { Soldier-worker (reproductives) }\end{array}$ & $\begin{array}{l}\text { Hughes et al, } 2003 \\
\text { Johnson \& Tsutsui, } 2011 \\
\text { He et al, } 2017 \\
\text { Wu et al, } 2018\end{array}$ \\
\hline
\end{tabular}


目蚜虫和缨翅目蓟马的研究还很欠缺, 关于其行为 分化相关机制的研究更是胗䇣无几。与行为分化相 关的表型可塑性是社会性昆虫的一个主要特征, 虽 然不同的社会性昆虫是独立进化的 (Anderson, 1984), 但通过对主要社会性昆虫的比较分析揭示 了一些与级型分化有关的保守基因和信号通路 (Berens et al, 2015), 包括胰岛素信号通路、保幼激 素和蜕皮激素信号通路、生物胺、卵黄原蛋白等 (Corona et al, 2016)。这些相对保守的基因或信号通 路在不同社会性昆虫类群中是否具有一致的调控 作用, 以及是否参与调控蚜虫和蓟马社会性行为的 分化, 尚有待开展深入的研究。除了膜翅目和蜚蠊 目常见社会性昆虫外, 将来对更多社会性昆虫类群 行为分化机制的研究将有助于我们更好地理解昆 虫纲中社会性行为的起源和演化过程。

随着新一代的测序技术和生物信息学工具的 日益发展和普及, 以及比较基因组和转录组学、表 观遗传学、RNA干扰及基因编辑技术等的广泛应用, 越来越多的社会性昆虫的基因组将被破译, 通过对 不同社会性昆虫类群的比较基因组和转录组学研 究工作的开展, 将为确定参与调控社会性昆虫行为 分化的基因或调控网络以及揭示社会性昆虫行为 分化的调控机理提供更多的线索。

\section{ORCID}

张慧 (D) https://orcid.org/0000-0002-4883-1642

黄晓否 (D) https://orcid.org/0000-0002-6839-9922

\section{参考文献}

Ament SA, Corona M, Pollock HS, Robinson GE (2008) Insulin signaling is involved in the regulation of worker division of labor in honey bee colonies. Proceedings of the National Academy of Sciences, USA, 105, 4226-4231.

Ament SA, Velarde RA, Kolodkin MH, Moyse D, Robinson GE (2011) Neuropeptide Y-like signalling and nutritionally mediated gene expression and behaviour in the honey bee. Insect Molecular Biology, 20, 335-345.

Amsalem E, Malka O, Grozinger C, Hefetz A (2014) Exploring the role of juvenile hormone and vitellogenin in reproduction and social behavior in bumble bees. BMC Evolutionary Biology, 14, 45.

Anderson KE, Gadau J, Mott BM, Johnson RA, Altamirano A, Strehl C, Fewell JH (2006) Distribution and evolution of genetic caste determination in Pogonomyrmex seedharvester ants. Ecology, 87, 2171-2184.

Anderson M (1984) The evolution of eusociality. Annual Review of Ecology and Systematics, 15, 165-189.
Aonuma H, Watanabe T (2012) Octopaminergic system in the brain controls aggressive motivation in the ant, Formica japonica. Acta Biologica Hungarica, 63, 63-68.

Barron AB, Maleszka R, Vander Meer RK, Robinson GE (2007) Octopamine modulates honey bee dance behavior. Proceedings of the National Academy of Sciences, USA, 104, 1703-1707.

Barron AB, Oldroyd BP (2001) Social regulation of ovary activation in "anarchistic" honey-bees (Apis mellifera). Behavioral Ecology and Sociobiology, 49, 214-219.

Berens AJ, Hunt JH, Toth AL (2015) Comparative transcriptomics of convergent evolution: Different genes but conserved pathways underlie caste phenotypes across lineages of eusocial insects. Molecular Biology and Evolution, 32, 690-703.

Bonasio R, Li QY, Lian JM, Mutti NS, Jin LJ, Zhao HM, Zhang P, Wen P, Xiang H, Ding Y, Jin ZH, Shen SS, Wang ZJ, Wang W, Wang J, Berger SL, Liebig J, Zhang GJ, Reinberg D (2012) Genome-wide and caste-specific DNA methylomes of the ants Camponotus floridanus and Harpegnathos saltator. Current Biology, 22, 1755-1764.

Bonasio R, Zhang GJ, Ye CY, Mutti NS, Fang XD, Qin N, Donahue G, Yang PC, Li QY, Li C, Zhang P, Huang ZY, Berger SL, Reinberg D, Wang J, Liebig J (2010) Genomic comparison of the ants Camponotus floridanus and Harpegnathos saltator. Science, 329, 1068-1071.

Brian MV, Hibble J (1964) Studies of caste differentiation in Myrmica rubra L. Insectes Sociaux, 11, 223-238.

Cahan SH, Keller L (2003) Complex hybrid origin of genetic caste determination in harvester ants. Nature, 424, 306-309.

Cassill DL, Tschinkel WR (1999) Task selection by workers of the fire ant Solenopsis invicta. Behavioral Ecology and Sociobiology, 45, 301-310.

Chandra V, Fetter-Pruneda I, Oxley PR, Ritger AL, Mckenzie SK, Libbrecht R, Kronauer DJC (2018) Social regulation of insulin signaling and the evolution of eusociality in ants. Science, 361, 398-402.

Chandrasekaran S, Ament SA, Eddy JA, Rodriguez-Zas SL, Schatz BR, Price ND, Robinson GE (2011) Behavior-specific changes in transcriptional modules lead to distinct and predictable neurogenomic states. Proceedings of the National Academy of Sciences, USA, 108, 18020-18025.

Chittka A, Wurm Y, Chittka L (2012) Epigenetics: The making of ant castes. Current Biology, 22, R835-R838.

Cornette R, Gotoh H, Koshikawa S, Miura T (2008) Juvenile hormone titers and caste differentiation in the damp-wood termite Hodotermopsis sjostedti (Isoptera, Termopsidae). Journal of Insect Physiology, 54, 922-930.

Corona M, Libbrecht R, Wheeler DE (2016) Molecular mechanisms of phenotypic plasticity in social insects. Current Opinion in Insect Science, 13, 55-60.

Corona M, Libbrecht R, Wurm Y, Riba-Grognuz O, Studer RA, Keller L (2013) Vitellogenin underwent subfunctionali- 
zation to acquire caste and behavioral specific expression in the harvester ant Pogonomyrmex barbatus. PLoS Genetics, 9, e1003730.

Crespi BJ (1992) Eusociality in Australian gall thrips. Nature, 359, 724-726.

Daugherty THF, Toth AL, Robinson GE (2011) Nutrition and division of labor: Effects on foraging and brain gene expression in the paper wasp Polistes metricus. Molecular Ecology, 20, 5337-5347.

de Azevedo SV, Hartfelder K (2008) The insulin signaling pathway in honey bee (Apis mellifera) caste development-Differential expression of insulin-like peptides and insulin receptors in queen and worker larvae. Journal of Insect Physiology, 54, 1064-1071.

Dong SZ, Ye GY, Guo JY, Hu C (2009) Roles of ecdysteroid and juvenile hormone in vitellogenesis in an endoparasitic wasp, Pteromalus puparum (Hymenoptera: Pteromalidae). General and Comparative Endocrinology, 160, 102-108.

Fei HX, Henderson G (2002) Formosan subterranean termite (Isoptera: Rhinotermitidae) wood consumption and worker survival as affected by temperature and soldier proportion. Environmental Entomology, 31, 509-514.

Ferreira PG, Patalano S, Chauhan R, Ffrench-Constant R, Gabaldón T, Guigó R, Sumner S (2013) Transcriptome analyses of primitively eusocial wasps reveal novel insights into the evolution of sociality and the origin of alternative phenotypes. Genome Biology, 14, R20.

Flatt T, Tu MP, Tatar M (2005) Hormonal pleiotropy and the juvenile hormone regulation of Drosophila development and life history. BioEssays, 27, 999-1010.

Frumhoff PC, Baker J (1988) A genetic component to division of labour within honey bee colonies. Nature, 333, 358-361.

Gadagkar R, Gordon D, Keller L, Michod R, Queller D, Robinson GE, Strassmann J, West-Eberhard MJ (2019) Insights and opportunities in insect social behavior. Current Opinion in Insect Science, 34, ix-xx.

Greene MJ, Gordon DM (2003) Social insects: Cuticular hydrocarbons inform task decisions. Nature, 423, 32.

Harpur BA, Kent CF, Molodtsova D, Lebon JMD, Alqarni AS, Owayss AA, Zayed A (2014) Population genomics of the honey bee reveals strong signatures of positive selection on worker traits. Proceedings of the National Academy of Sciences, USA, 111, 2614-2619.

Harrison MC, Jongepier E, Robertson HM, Arning N, Bitard-Feildel T, Chao $\mathrm{H}$, Childers CP, Dinh $\mathrm{H}$, Doddapaneni H, Dugan S, Gowin J, Greiner C, Han Y, Hu HF, Hughes DST, Huylmans AK, Kemena C, Kremer LPM, Lee SL, Lopez-Ezquerra A, Mallet L, Monroy-Kuhn JM, Moser A, Murali SC, Muzny DM, Otani S, Piulachs MD, Poelchau M, Qu JX, Schaub F, Wada-Katsumata A, Worley KC, Xie QL, Ylla G, Poulsen M, Gibbs RA, Schal C, Richards S, Belles X, Korb J, Bornberg-Bauer E (2018) Hemimetabolous genomes reveal molecular basis of termite eusociality. Nature Ecology \& Evolution, 2, 557-566.
Hayashi Y, Lo N, Miyata H, Kitade O (2007) Sex-linked genetic influence on caste determination in a termite. Science, 318, 985-987.

Haydak MH (1943) Larval food and development of castes in the honeybee. Journal of Economic Entomology, 36, 778-792.

He XJ, Zhou LB, Pan QZ, Barron AB, Yan WY, Zeng ZJ (2017) Making a queen: An epigenetic analysis of the robustness of the honeybee (Apis mellifera) queen developmental pathway. Molecular Ecology, 26, 1598-1607.

Hughes WOH, Sumner S, Van Borm S, Boomsma JJ (2003) Worker caste polymorphism has a genetic basis in Acromyrmex leaf-cutting ants. Proceedings of the National Academy of Sciences, USA, 100, 9394-9397.

Ishikawa Y, Aonuma H, Sasaki K, Miura T (2016) Tyraminergic and octopaminergic modulation of defensive behavior in termite soldier. PLoS ONE, 11, e0154230.

Jaffé R, Kronauer DJ, Kraus FB, Boomsma JJ, Moritz RF (2007) Worker caste determination in the army ant Eciton burchellii. Biology Letters, 3, 513-516.

Johnson BR, Tsutsui ND (2011) Taxonomically restricted genes are associated with the evolution of sociality in the honey bee. BMC Genomics, 12, 164.

Judd TM, Teal PEA, Hernandez EJ, Choudhury T, Hunt JH (2015) Quantitative differences in nourishment affect caste-related physiology and development in the paper wasp Polistes metricus. PLoS ONE, 10, e0116199.

Julian GE, Fewell JH, Gadau J, Johnson RA, Larrabee D (2002) Genetic determination of the queen caste in an ant hybrid zone. Proceedings of the National Academy of Sciences, USA, 99, 8157-8160.

Kaatz H, Eichmüller S, Kreissl S (1994) Stimulatory effect of octopamine on juvenile hormone biosynthesis in honey bees (Apis mellifera): Physiological and immunocytochemical evidence. Journal of Insect Physiology, 40, 865-872.

Kamakura M (2011) Royalactin induces queen differentiation in honeybees. Nature, 473, 478-483.

Kamhi JF, Nunn K, Robson SKA, Traniello JFA (2015) Polymorphism and division of labour in a socially complex ant: Neuromodulation of aggression in the Australian weaver ant, Oecophylla smaragdina. Proceedings of the Royal Society B: Biological Sciences, 282, 20150704.

Kapheim KM, Pan H, Li C, Salzberg SL, Puiu D, Magoc T, Robertson HM, Hudson ME, Venkat A, Fischman BJ, Hernandez A, Yandell M, Ence D, Holt C, Yocum GD, Kemp WP, Bosch J, Waterhouse RM, Zdobnov EM, Stolle E, Kraus FB, Helbing S, Moritz RFA, Glastad KM, Hunt BG, Goodisman MAD, Hauser F, Grimmelikhuijzen CJP, Pinheiro DG, Nunes FMF, Soares MPM, Tanaka ÉD, Simões ZLP, Hartfelder K, Evans JD, Barribeau SM, Johnson RM, Massey JH, Southey BR, Hasselmann M, Hamacher D, Biewer M, Kent CF, Zayed A, Blatti C, Sinha S, Johnston JS, Hanrahan SJ, Kocher SD, Wang J, Robinson GE, Zhang G (2015) Genomic signatures of evolutionary 
transitions from solitary to group living. Science, 348, 1139-1143.

Kocher SD, Pellissier L, Veller C, Purcell J, Nowak MA, Chapuisat M, Pierce NE (2014) Transitions in social complexity along elevational gradients reveal a combined impact of season length and development time on social evolution. Proceedings of the Royal Society B: Biological Sciences, 281, 20140627.

Korb J (2015) Juvenile hormone: A central regulator of termite caste polyphenism. Advances in Insect Physiology, 48, 131-161.

Korb J, Belles X (2017) Juvenile hormone and hemimetabolan eusociality: A comparison of cockroaches with termites. Current Opinion in Insect Science, 22, 109-116.

Korb J, Schmidinger S (2004) Help or disperse? Cooperation in termites influenced by food conditions. Behavioral Ecology and Sociobiology, 56, 89-95.

LeBoeuf AC, Benton R, Keller L (2013) The molecular basis of social behavior: Models, methods and advances. Current Opinion in Neurobiology, 23, 3-10.

Li BB, Hou L, Zhu D, Xu XL, An SH, Wang XH (2018) Identification and caste-dependent expression patterns of DNA methylation associated genes in Bombus terrestris. Scientific Reports, 8, 2332.

Lucas C, Sokolowski MB (2009) Molecular basis for changes in behavioral state in ant social behaviors. Proceedings of the National Academy of Sciences, USA, 106, 6351-6356.

Marco Antonio DS, Guidugli-Lazzarini KR, do Nascimento AM, Simões ZLP, Hartfelder K (2008) RNAi-mediated silencing of vitellogenin gene function turns honeybee (Apis mellifera) workers into extremely precocious foragers. Naturwissenschaften, 95, 953-961.

Marshall H, Lonsdale ZN, Mallon EB (2019) Methylation and gene expression differences between reproductive and sterile bumblebee workers. Evolution Letters, 3, 485-499.

Masuoka Y, Yaguchi H, Toga K, Shigenobu S, Maekawa K (2018) TGF $\beta$ signaling related genes are involved in hormonal mediation during termite soldier differentiation. PLoS Genetics, 14, e1007338.

Matsuura K, Himuro C, Yokoi T, Yamamoto Y, Vargo EL, Keller L (2010) Identification of a pheromone regulating caste differentiation in termites. Proceedings of the National Academy of Sciences, USA, 107, 12963-12968.

Mizunami M, Yamagata N, Nishino H (2010) Alarm pheromone processing in the ant brain: An evolutionary perspective. Frontiers in Behavioral Neuroscience, 4, 28.

Monastirioti M (1999) Biogenic amine systems in the fruit fly Drosophila melanogaster. Microscopy Research and Technique, 45, 106-121.

Mott CM, Breed MD (2012) Insulin modifies honeybee worker behavior. Insects, 3, 1084-1092.

Nelson CM, Ihle KE, Fondrk MK, Page RE, Amdam GV (2007) The gene vitellogenin has multiple coordinating effects on social organization. PLoS Biology, 5, e62.
Norman VC, Hughes WOH (2016) Behavioural effects of juvenile hormone and their influence on division of labour in leaf-cutting ant societies. Journal of Experimental Biology, 219, 8-11.

Nouvian M, Mandal S, Jamme C, Claudianos C, d'Ettorre P, Reinhard J, Barron AB, Giurfa M (2018) Cooperative defence operates by social modulation of biogenic amine levels in the honey bee brain. Proceedings of the Royal Society B: Biological Sciences, 285, 20172653.

Page RE, Erickson EH (1988) Reproduction by worker honey bees (Apis mellifera L.). Behavioral Ecology and Sociobiology, 23, 117-126.

Pearcy M, Aron S, Doums C, Keller L (2004) Conditional use of sex and parthenogenesis for worker and queen production in ants. Science, 306, 1780-1783.

Rehan SM, Berens AJ, Toth AL (2014) At the brink of eusociality: Transcriptomic correlates of worker behaviour in a small carpenter bee. BMC Evolutionary Biology, 14, 260.

Reim T, Scheiner R (2014) Division of labour in honey bees: Age- and task-related changes in the expression of octopamine receptor genes. Insect Molecular Biology, 23, 833-841.

Robinson GE (1992) Regulation of division of labor in insect societies. Annual Review of Entomology, 37, 637-665.

Robinson GE, Page RE (1988) Genetic determination of guarding and undertaking in honey-bee colonies. Nature, 333, 356-358.

Scheiner R, Baumann A, Blenau W (2006) Aminergic control and modulation of honeybee behaviour. Current Neuropharmacology, 4, 259-276.

Schulz DJ, Sullivan JP, Robinson GE (2002) Juvenile hormone and octopamine in the regulation of division of labor in honey bee colonies. Hormones and Behavior, 42, 222-231.

Schwander T, Humbert JY, Brent CS, Cahan SH, Chapuis L, Renai E, Keller L (2008) Maternal effect on female caste determination in a social insect. Current Biology, 18, 265-269.

Sheehan MJ, Botero CA, Hendry TA, Sedio BE, Jandt JM, Weiner S, Toth AL, Tibbetts EA (2015) Different axes of environmental variation explain the presence vs. extent of cooperative nest founding associations in Polistes paper wasps. Ecology Letters, 18, 1057-1067.

Simola DF, Graham RJ, Brady CM, Enzmann BL, Desplan C, Ray A, Zwiebel LJ, Bonasio R, Reinberg D, Liebig J, Berǵer SL (2016) Epigenetic (re)programming of caste-specific behavior in the ant Camponotus floridanus. Science, 351, aac6633.

Simola DF, Wissler L, Donahue G, Waterhouse RM, Helmkampf M, Roux J, Nygaard S, Glastad KM, Hagen DE, Viljakainen L, Reese JT, Hunt BG, Graur D, Elhaik E, Kriventseva EV, Wen J, Parker BJ, Cash E, Privman E, Childers CP, Muñoz-Torres MC, Boomsma JJ, Bornberg-Bauer E, Currie CR, Elsik CG, Suen G, 
Goodisman MAD, Keller L, Liebig J, Rawls A, Reinberg D, Smith CD, Smith CR, Tsutsui N, Wurm Y, Zdobnov EM, Berger SL, Gadau J (2013) Social insect genomes exhibit dramatic evolution in gene composition and regulation while preserving regulatory features linked to sociality. Genome Research, 23, 1235-1247.

Slessor KN, Kaminski LA, King GGS, Borden JH, Winston ML (1988) Semiochemical basis of the retinue response to queen honey bees. Nature, 332, 354-356.

Smith CR, Toth AL, Suarez AV, Robinson GE (2008) Genetic and genomic analyses of the division of labour in insect societies. Nature Reviews Genetics, 9, 735-748.

Steller MM, Kambhampati S, Caragea D (2010) Comparative analysis of expressed sequence tags from three castes and two life stages of the termite Reticulitermes flavipes. BMC Genomics, 11, 463.

Stern DL, Foster WA (1996) The evolution of soldiers in aphids. Biological Reviews, 71, 27-79.

Sullivan JP, Jassim O, Fahrbach SE, Robinson GE (2000) Juvenile hormone paces behavioral development in the adult worker honey bee. Hormones and Behavior, 37, 1-14.

Sumner S (2014) The importance of genomic novelty in social evolution. Molecular Ecology, 23, 26-28.

Szczuka A, Korczyńska J, Wnuk A, Symonowicz B, Gonzalez-Szwacka A, Mazurkiewicz P, Kostowski W, Godzińska EJ (2013) The effects of serotonin, dopamine, octopamine and tyramine on behavior of workers of the ant Formica polyctena during dyadic aggression tests. Acta Neurobiologiae Experimentalis, 73, 495-520.

Tarver MR, Florane CB, Zhang DH, Grimm C, Lax AR (2012) Methoprene and temperature effects on caste differentiation and protein composition in the Formosan subterranean termite, Coptotermes formosanus. Journal of Insect Science, $12,18$.

Terrapon N, Li C, Robertson HM, Ji L, Meng XH, Booth W, Chen ZS, Childers CP, Glastad KM, Gokhale K, Gowin J, Gronenberg W, Hermansen RA, Hu HF, Hunt BG, Huylmans AK, Khalil SMS, Mitchell RD, Munoz-Torres MC, Mustard JA, Pan HL, Reese JT, Scharf ME, Sun FM, Vogel H, Xiao J, Yang W, Yang ZK, Yang ZQ, Zhou JJ, Zhu JW, Brent CS, Elsik CG, Goodisman MAD, Liberles DA, Roe RM, Vargo EL, Vilcinskas A, Wang J, Bornberg-Bauer E, Korb J, Zhang GJ, Liebig J (2014) Molecular traces of alternative social organization in a termite genome. Nature Communications, 5, 3636.

Toth AL, Kantarovich S, Meisel AF, Robinson GE (2005) Nutritional status influences socially regulated foraging ontogeny in honey bees. Journal of Experimental Biology, 208, 4641-4649.

Toth AL, Rehan SM (2017) Molecular evolution of insect sociality: An eco-evo-devo perspective. Annual Review of Entomology, 62, 419-442.

Toth AL, Robinson GE (2007) Evo-devo and the evolution of social behavior. Trends in Genetics, 23, 334-341.
Trible W, Kronauer DJC (2017) Caste development and evolution in ants: It's all about size. Journal of Experimental Biology, 220, 53-62.

Wada-Katsumata A, Yamaoka R, Aonuma H (2011) Social interactions influence dopamine and octopamine homeostasis in the brain of the ant Formica japonica. Journal of Experimental Biology, 214, 1707-1713.

Watanabe D, Gotoh H, Miura T, Maekawa K (2014) Social interactions affecting caste development through physiological actions in termites. Frontiers in Physiology, 5, 127.

Weiner SA, Toth AL (2012) Epigenetics in social insects: A new direction for understanding the evolution of castes. Genetics Research International, 2012, 609810.

Weitekamp CA, Libbrecht R, Keller L (2017) Genetics and evolution of social behavior in insects. Annual Review of Genetics, 51, 219-239.

Wheeler D (1996) The role of nourishment in oogenesis. Annual Review of Entomology, 41, 407-431.

Whitfield CW, Cziko AM, Robinson GE (2003) Gene expression profiles in the brain predict behavior in individual honey bees. Science, 302, 296-299.

Wiernasz DC, Cole BJ (2010) Patriline shifting leads to apparent genetic caste determination in harvester ants. Proceedings of the National Academy of Sciences, USA, 107, 12958-12962.

Wilson EO (1971) The Insect Societies. Harvard University Press, Cambridge.

Wilson EO (1985) The sociogenesis of insect colonies. Science, 228, 1489-1495.

Wilson EO (1987) Causes of ecological success: The case of the ants. Journal of Animal Ecology, 56, 1-9.

Wilson EO, Hölldobler B (2005) Eusociality: Origin and consequences. Proceedings of the National Academy of Sciences, USA, 102, 13367-13371.

Wissler L, Gadau J, Simola DF, Helmkampf M, Bornberg-Bauer E (2013) Mechanisms and dynamics of orphan gene emergence in insect genomes. Genome Biology and Evolution, 5, 439-455.

Woodard SH, Bloch GM, Band MR, Robinson GE (2014) Molecular heterochrony and the evolution of sociality in bumblebees (Bombus terrestris). Proceedings of the Royal Society B: Biological Sciences, 281, 20132419.

Wu Q, Brown MR (2006) Signaling and function of insulin-like peptides in insects. Annual Review of Entomology, 51, 1-24.

Wu T, Dhami GK, Thompson GJ (2018) Soldier-biased gene expression in a subterranean termite implies functional specialization of the defensive caste. Evolution \& Development, 20, 3-16.

Zayed A, Kent CF (2015) Genomics, physiology and behaviour of social insects. Advances in Insect Physiology, 48, 1-363.

(责任编委: 周欣 责任编辑: 时意专) 